\title{
REVIEW ARTICLE OPEN First-principles calculations of lattice dynamics and thermal properties of polar solids
}

\author{
Yi Wang ${ }^{1}$, Shun-Li Shang ${ }^{1}$, Huazhi Fang ${ }^{1}$, Zi-Kui Liu ${ }^{1}$ and Long-Qing Chen ${ }^{1}$
}

Although the theory of lattice dynamics was established six decades ago, its accurate implementation for polar solids using the direct (or supercell, small displacement, frozen phonon) approach within the framework of density-function-theory-based first-principles calculations had been a challenge until recently. It arises from the fact that the vibration-induced polarization breaks the lattice periodicity, whereas periodic boundary conditions are required by typical first-principles calculations, leading to an artificial macroscopic electric field. The article reviews a mixed-space approach to treating the interactions between lattice vibration and polarization, its applications to accurately predicting the phonon and associated thermal properties, and its implementations in a number of existing phonon codes.

npj Computational Materials (2016) 2, 16006; doi:10.1038/npjcompumats.2016.6; published online 13 May 2016

\section{INTRODUCTION}

Lattice dynamics is the study of the collective atomic vibrations in a crystal. The concept of phonons was introduced by Tamm ${ }^{1}$ in 1930 through an observation of the particle-like energetics of atomic vibrations in a crystal, similar to the wave-particle duality in quantum mechanics. Lattice dynamics has since become an important branch of condensed matter physics and is critical for understanding the thermal properties of crystalline solids at finite temperatures. ${ }^{2,3}$ For example, the phonon densities of states are required for evaluating thermodynamic properties of a crystal, ${ }^{4-6}$ such as thermal expansion coefficients, heat capacity, entropy and lattice thermal conductivity. ${ }^{7-9}$ There exist excellent reference books on lattice dynamics, e.g., by Wallace ${ }^{10}$ and by Born and Huang. ${ }^{2}$

With the advances in density functional theory calculations, ${ }^{11-15}$ all the input data needed by lattice dynamics can now be obtained by the first-principles approach solely based on the crystal structure and atomic numbers. Currently, there are essentially two implementations in wide use for the firstprinciples calculations of lattice dynamics: the linear-response approach $^{16}$ and the direct approach. ${ }^{17,18} \mathrm{~A}$ general review of first-principles approach to phonon theory was given by Baroni et al. $^{16}$ focused on the linear-response approach. ${ }^{16,19,20}$

The linear-response approach directly evaluates the dynamical matrix at a predetermined reference coarse grid in the wavevector space through the density functional perturbation theory. ${ }^{16,19,20}$ Then the backward Fourier transform of the calculated dynamical matrix at the coarse wave-vector grid is employed to extract the interatomic force constants on the corresponding real-space grid. In contrast, the direct approach first calculates the force constants using a predetermined reference supercell of the primitive cell. In the literature, the direct approach $^{4,16,17,21,22}$ is also referred as the supercell method, the small-displacement method or the frozen-phonon approach. The features of the linear-response approach and the direct approach are compared in Table 1. A collection of phonon/first-principles codes, including $\mathrm{YPHON}^{23}{ }^{23}$ ShengBTE, ${ }^{8}$ PhonTS, $^{7}$ Phonopy, ${ }^{18}$ ALAMODE, ${ }^{24}$ PHON, ${ }^{21}$ ATAT, ${ }^{4,22}$ PHONON, ${ }^{17,25-27}$ PWSCF/ QUANTUM ESPRESSO, ${ }^{14}$ ABINIT, ${ }^{15}$ CASTEP, ${ }^{13}$ CRYSTAL ${ }^{28}$ and VASP (the Vienna $A b$ initio Simulation Package) ${ }^{11,12}$ that can be employed to calculate phonon and related properties are briefed in Table 2.

The present review focuses on the theory of lattice dynamics for polar solids. Here a polar solid implies an insulator or a semiconductor composed of cations with positive charges and anions with negative charges. As a matter of fact, the majorities of modern functional materials are made of polar solids, such as the topological crystalline insulator group-IV tellurides, ${ }^{29}$ the ferroelectrics and multiferroics, ${ }^{30}$ and materials for solar cells. ${ }^{31}$ The accurate descriptions of phonon properties have key roles for the understandings and developments of these materials.

For certain optical atomic vibration modes, cations and anions vibrate in opposite directions creating dipole-dipole interactions and hence homogeneous electric fields, which have to be treated with caution in phonon calculations. As an illustration, Figure 1 shows the effects of vibration-induced polarization within supercells. The supercell described in Figure 1a is commensurate with the wavelength of the lattice vibration of interest, so the averaged electric polarization is zero, and thus no macroscopic electric field is generated. It should be pointed out that there are still internal dipole-dipole interactions within an individual supercell, but they are already accounted for in first-principles calculations of the interatomic force constants. ${ }^{32,33}$ In Figure $1 \mathrm{~b}$, the supercell is incommensurate with the wavelength of a lattice vibration, and the corresponding phonon produces a nonzero-averaged electric polarization and thus a nonzero artificial macroscopic electric field. Figure 1c shows how the artificial electric polarization varies with the supercell size (or the supercell geometry for the threedimentional case). In existing literature, ${ }^{2,34}$ polar effects were mainly discussed in the long-wavelength limit. It has been shown that the homogeneous field presents significant difficulties in calculating the phonon frequencies of polar materials for the

\footnotetext{
${ }^{1}$ Department of Materials Science and Engineering, The Pennsylvania State University, University Park, Pennsylvania, PA, USA. 
Table 1. The linear-response approach versus the direct approach

\begin{tabular}{|c|c|c|}
\hline Features & Linear response approach & Direct approach \\
\hline Disadvantage & $\begin{array}{l}\text { Extensive programming required and it sometimes has } \\
\text { special requirements for the form of pseudopotential in } \\
\text { DFT calculations }\end{array}$ & $\begin{array}{l}\text { Phonon frequencies can be accurately calculated } \\
\text { only at q points that are commensurate with } \\
\text { supercell geometry }\end{array}$ \\
\hline $\begin{array}{l}\text { Additional calculations for polar } \\
\text { materials }\end{array}$ & $\begin{array}{l}\text { Separate calculation of dynamical matrix at } \Gamma \text { point, } \\
\text { Born effective charges and dielectric constants; } \\
\text { additional calculation to separate coulombic } \\
\text { contribution from dynamical matrix }\end{array}$ & $\begin{array}{l}\text { Separate calculation of Born effective charges and } \\
\text { dielectric constants }\end{array}$ \\
\hline $\begin{array}{l}\text { For accurate phonon dispersions or } \\
\text { density of states for polar solids }\end{array}$ & $\begin{array}{l}\text { Forward Fourier interpolation using the interatomic } \\
\text { force constants followed by adding the coulombic } \\
\text { contribution back. }\end{array}$ & $\begin{array}{l}\text { Adding coulombic contribution as a constant term } \\
\text { to the interatomic force constants followed by } \\
\text { forward Fourier interpolation }\end{array}$ \\
\hline
\end{tabular}

Table 2. A collection of phonon/first-principles codes

\begin{tabular}{|c|c|c|}
\hline Codes & Abilities & $\begin{array}{l}\text { Method to compute polar } \\
\text { effects on phonons }\end{array}$ \\
\hline PWSCF/QUANTUM ESPRESSO ${ }^{14}$ & Electronic structure; phonon & Linear-response approach \\
\hline CASTEP $^{13}$ & Electronic structure; phonon & Linear-response approach \\
\hline CRYSTAL $^{28}$ & Electronic structure; phonon & Mixed-space approach \\
\hline VASP $^{11,12}$ & Electronic structure; phonon & Not available \\
\hline $\mathrm{YPHON}^{23}$ & Phonon & Mixed-space approach \\
\hline Phonopy ${ }^{18}$ & Phonon; thermal conductivity; thermodynamic properties & Mixed-space approach \\
\hline ALAMODE $^{24}$ & $\begin{array}{l}\text { Phonon; thermal conductivity; thermodynamic properties; } \\
\text { anharmonicity }\end{array}$ & Mixed-space approach \\
\hline PHONON $^{17,25-27}$ & Phonon; thermodynamic properties & Only accurate at the $\Gamma$ point \\
\hline $\mathrm{PHON}^{21}$ & Phonon; thermodynamic properties & Not available \\
\hline ATAT $^{4,22}$ & Phonon; thermodynamic properties & Not available \\
\hline
\end{tabular}

direct approach under the Born-von Kármán boundary conditions. $^{35}$

In statistical thermodynamics, a phonon represents a quantized vibrational mode characterized by a frequency at a given reciprocal lattice wave-vector point. ${ }^{2,10}$ An accurate thermodynamic calculation ${ }^{4-6}$ requires the phonon frequency distribution, i.e., the phonon density of states, calculated over a fine mesh in the wave-vector space. Although in principle the phonon frequencies at any wave-vector points can be calculated by the first-principles approach, it is still computationally too expensive to account for all mesh points in the wave-vector space. To reduce the computational cost, a practical approach is to first calculate either the force constants for a predefined supercell in the real space or the dynamical matrix for a predefined grid in the reciprocal space, ${ }^{16}$ and followed by Fourier interpolations to evaluate phonon properties at arbitrary wave-vector points. For polar solids, such a strategy has only been implemented in the linear-response approach until recently when a mixed-space method becomes available. ${ }^{21,36,37}$ The mixed-space approach makes it possible to accurately calculate the phonon properties for polar solids within the framework of direct approach for phonon calculations. ${ }^{9,28}$ The mixed-space approach has also been extended to lattice thermal conductivity calculations ${ }^{7-9,38}$ where the third-order force constants are needed.

By this review, we will show that (i) the Born-von Kármán boundary conditions ${ }^{39}$ still apply when the phonon frequencies are calculated at the exact wave-vector points; ${ }_{i}^{40}$ (ii) The effects of vibration-induced polarization on phonons at an arbitrary wave-vector point can be understood in the real space; and (iii) The longitudinal optical-transverse optical (LO-TO) phonon splitting ${ }^{34,40}$ can be predicted accurately at the exact wave-vector points by the direct approach without explicitly handling the effects of macroscopic electric fields.

The present paper is organized as follows: 'Basic lattice dynamics of polar solids' describes the basics of lattice dynamics and the fundamentals of the mixed-space approach. 'Helmholtz energy and quasiharmonic approximation' outlines the first-principles thermodynamics based on the phonon theory; More discussions of the mixed-space approach are given in 'The mixed-space approach'. 'Computational procedure' summarizes the common procedures in phonon calculations; 'Phonon software packages have implemented the mixed-space approach' briefs the implementation of the mixed-space approach in several software packages. Extensive applications of the mixed-space approach are summarized in 'Recent calculations using the mixed-space approach'. 'Other phonon software packages' briefs a list of other phonon codes implemented differently from the mixed-space approach for polar solids. 'Software packages for both electronic and phonon calculations' introduces a few widely in use first-principles codes for both electronic and phonon calculations. Finally, the last section is the 'Summary'. 

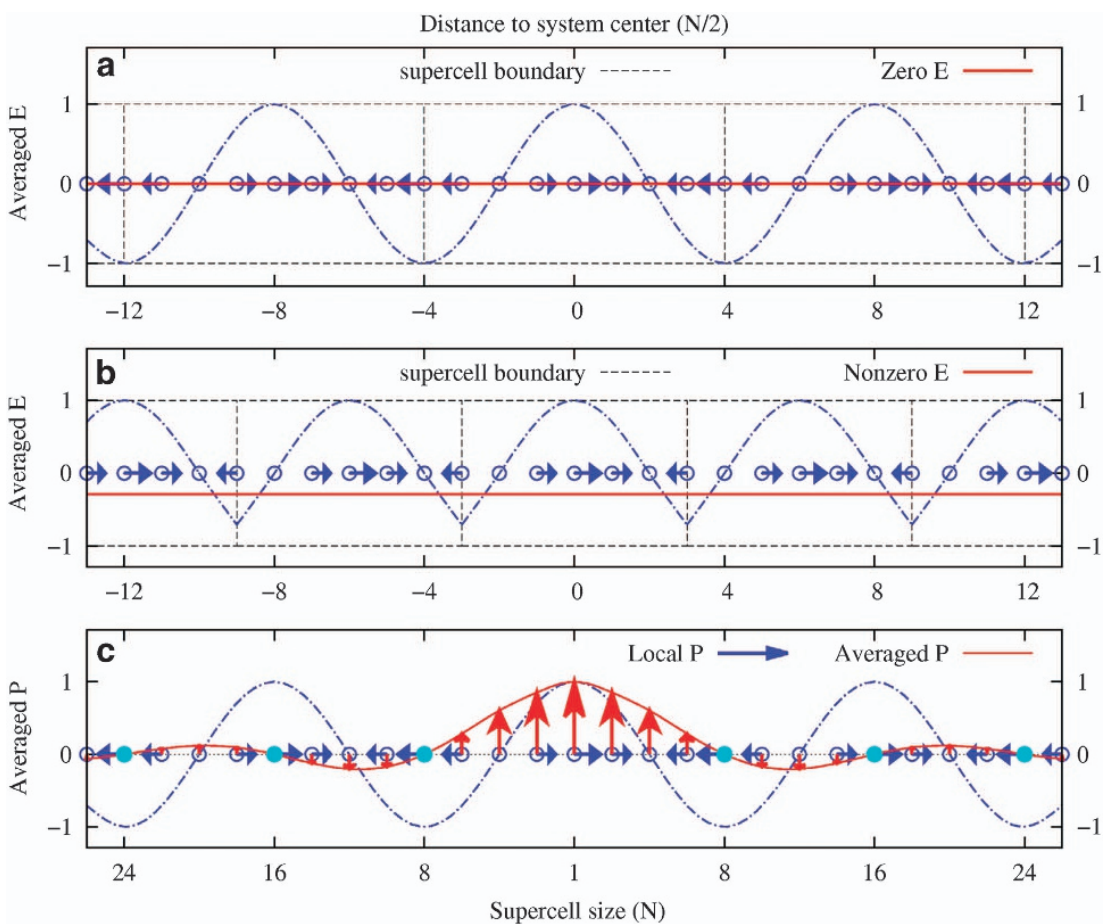

Figure 1. Vibration-induced polarization at a selected wave-vector point $q=2 \pi(1 / 8,0,0)$. The horizontal (solid blue) arrows indicate the local electric polarization of a primitive unit cell due to the optical vibration. The cosine type (blue dot-dashed) curves are used as a guide to eyes for the periodicity of electric polarization. The open (blue) circles mark the positions of primitive unit cells. The dashed (black) lines in (a,b) designate the supercell boundaries. (a) The supercell geometry (supercell size $N=8$ ) is commensurate with the wave-vector, so the averaged electric polarization is zero, and no macroscopic electric field is generated. (b) The supercell (supercell size $N=6$ ) is incommensurate with the wave-vector, and therefore, the average electric polarization is nonzero, creating an artificial macroscopic electric field. (c) The variation of the averaged electric polarization with supercell size is shown by the vertical (solid red) arrows enveloped by the solid (red) curve. The solid (cyan) circles in c mark the supercell sizes by which the averaged polarizations are zero.

\section{BASIC LATTICE DYNAMICS OF POLAR SOLIDS}

As phonons represent waves of collective atomic vibrations in a periodic pattern, we can choose to model a system by a repeated parallelepiped, i.e., a supercell. To formulate atomic vibrations within such a supercell, it is convenient to start from equations of motion for the atoms. For a polar solid, the forces can be divided into two additive contributions-analytic and nonanalytic. ${ }^{20}$ The terms analytic and nonanalytic can be traced back to the mathematical definition ${ }^{41}$ that a function is called analytic if and only if its Taylor series expansion about a reference point converges to the function at each point in some neighbourhood of the reference point, otherwise it is nonanalytic. Under the framework of first-principles calculations, the analytic contribution accounts for all the forces under the restricted periodic boundary conditions under which the averaged electric field is assumed to be zero. The nonanalytic contribution accounts the additional forces owing to a nonzero-averaged electric field.

The classical Newton's second law of motion for describing the atomic vibrations of a polar solid is given by

$$
m_{j} \frac{\partial^{2} u_{a}(t, j ; P)}{\partial t^{2}}=-\frac{\partial E(\mathbf{U})}{\partial u_{a}(t, j ; P)}+e \mathbf{Z}_{a}(j) \cdot \mathbf{E} .
$$

where $m_{j}$ represents the atomic mass of the $j$ th atom in the primitive unit cell, $t$, the time, the $a(a=\mathrm{x}, \mathrm{y}, \mathrm{z})$ component of the atomic displacement from its equilibrium position of the $j$ th atom in the Pth reference primitive unit cell within a supercell. The first term in the right-hand side of Equation (1) accounts for the analytic force due to the short-range interatomic interaction, where $\mathbf{U}$ represent the whole set of atomic displacements and $E(\mathbf{U})$ the total energy. The second term in the right-hand side of
Equation (1), with $-e$ the electron charge, accounts for the nonanalytic force due to the long-range coulombic interactions shown as the dot product between the Born effective charge ( $\mathbf{Z}$, a second-rank tensor, i.e., $3 \times 3$ matrix) and the averaged electric field (E) induced by the atomic vibration. The Born effective charges can be calculated as the change in electric polarization divided by the amount an ion is displaced. ${ }^{20,42}$

The analytic contribution in the Harmonic approximation

Within the harmonic approximation, one can truncate the first term $E(\mathbf{U})$ in the right-hand side of Equation (1) at the second order in Taylor series expansion

$$
E(\mathbf{U})=\frac{1}{2} \sum_{P, Q}^{N} \sum_{j, k}^{N_{P}} \sum_{a, \beta}^{3} \Phi_{a \beta}^{j k}(P, Q) u_{a}(t, j ; P) u_{\beta}(t, k ; Q),
$$

where $N$ represents the number of primitive unit cells contained in the supercell, $N_{P}$ the number of atoms in the primitive cell $P$, and $a$ and $\beta$ the Cartesian components $x, y$ and $z . \Phi_{\alpha \beta}^{j k}(P, Q)$ is the real-space interatomic force constant matrix representing the interactions between the $j$ th atom within the primitive unit cell $P$ and $k$ th atom within the primitive cell $Q$ under zero macroscopic electric field.

The nonanalytic contribution due to the vibration-induced electric field

The nonanalytic contribution accounts for the LO-TO splitting, i.e., the removal of degeneracy between the LO and TO phonons at the Brillouin zone center. ${ }^{35}$ In particular, the LO-TO splitting generally depends upon the direction along which the wave- 
vector approaches the Brillouin zone center (mainly for non-cubic solids), ${ }^{43}$ making the splitting discontinuous.

We now prove that the second term $e \mathbf{Z}_{a}(j) \cdot \mathbf{E}$ at the right-hand side of Equation (1) can be explicitly formulated at any wave-vector points, going beyond the long-wavelength limit by Cochran and Cowley. ${ }^{34}$ On the basis of the Born effective charge and atomic displacement, the averaged polarization ${ }^{44}$ can be evaluated by

$$
\mathbf{P}=\frac{e}{N V_{\mathrm{P}}} \sum_{k, Q}^{N_{p}, N} \mathbf{Z}(k) \cdot \mathbf{u}(t, k ; Q),
$$

where $V_{\mathrm{P}}$ is the volume of the primitive cell. The vibration-induced polarization is parallel to the direction of the wave-vector $\hat{\mathbf{q}}$ and can only have effects on the atomic vibrations along the direction of the wave-vector $\hat{\mathbf{q}}$. It is noticed that a normal mode with a wavevector $\mathbf{q}$ is nothing but a collective vibration of some parallel charged crystal planes with normal along $\hat{\mathbf{q}}$. As a result, the induced electric field by the lattice vibrations can be formulated $\mathrm{as}^{45}$

$$
\mathbf{E}=-\frac{4 \pi \mathbf{P}}{\hat{\mathbf{q}} \cdot \boldsymbol{\varepsilon}_{\infty} \cdot \hat{\mathbf{q}}}=-\frac{4 \pi e}{N V_{P}} \sum_{k, Q}^{N_{P}, N} \frac{\mathbf{Z}(k) \cdot \mathbf{u}(t, k ; Q)}{\hat{\mathbf{q}} \cdot \boldsymbol{\varepsilon}_{\infty} \cdot \hat{\mathbf{q}}},
$$

where $\hat{\mathbf{q}} \cdot \boldsymbol{\varepsilon}_{\infty} \cdot \hat{\mathbf{q}}$ represents a projection of the macroscopic dielectric constant tensor into $\hat{\mathbf{q}}$. As $\mathbf{E}$ is along $\hat{\mathbf{q}}$, we can also project $\mathbf{Z}_{a}(j)$ into $\hat{\mathbf{q}}$ in evaluating $e \mathbf{Z}_{\alpha}(j) \cdot \mathbf{E}$ and obtain

$$
e \mathbf{Z}_{a}(j) \cdot \mathbf{E}=-\frac{4 \pi e}{N V_{P}} \sum_{k, Q}^{N_{p}, N} \frac{[\mathbf{Z}(j) \cdot \hat{\mathbf{q}}]_{a}[\mathbf{Z}(k) \cdot \hat{\mathbf{q}}] \cdot \mathbf{u}(t, k ; Q)}{\hat{\mathbf{q}} \cdot \boldsymbol{\varepsilon}_{\infty} \cdot \hat{\mathbf{q}}}
$$

For a normal mode ${ }^{2,10}$ in which the atoms oscillate at the same frequency, $\omega$, in a periodic pattern, the displacements $\mathbf{u}(t, j ; P)$ can be expressed as

$$
u_{a}(t, k ; P)=u_{a}(j ; \mathbf{q}) \exp \{i \mathbf{q} \cdot[\mathbf{R}(P)+\mathbf{r}(j)-i \omega(\mathbf{q}) t]\},
$$

where $\mathbf{q}$ is wave-vector, $\mathbf{R}(P)$ the position of the $P$ th primitive cell in the supercell, and $\mathbf{r}(j)$ the position of the $j$ th atom in the primitive cell.

Then, inserting equation (6) into equation (5), we obtain

$$
\begin{aligned}
& e \mathbf{Z}_{\alpha}(j) \cdot \mathbf{E}=-\frac{4 \pi e}{N V_{P}} \sum_{k, Q}^{N_{p}, N} \sum_{\beta} \\
& \frac{[\mathbf{Z}(j) \cdot \hat{\mathbf{q}}]_{\alpha}[\mathbf{Z}(k) \cdot \hat{\mathbf{q}}]_{\beta} \cdot u_{\beta}(k ; \mathbf{q}) \exp \{i \mathbf{q} \cdot[\mathbf{R}(Q)+\mathbf{r}(k)]-i \omega(\mathbf{q}) t\}}{\hat{\mathbf{q}} \cdot \boldsymbol{\varepsilon}_{\infty} \cdot \hat{\mathbf{q}}} .
\end{aligned}
$$

The dynamical matrix

Substituting Equation (2) into Equation (1) together with utilizing Equation (7), the equation of motion for the atoms can be expressed in the reciprocal space, $\mathbf{q}$,

$$
-\omega^{2} w_{a}(j ; \mathbf{q})=-\sum_{k}^{N_{p}} \sum_{\beta}^{3} D_{\alpha \beta}^{j k}(\mathbf{q}) w_{\beta}(k ; \mathbf{q}),
$$

where we have replaced $u_{a}(j ; \mathbf{q})$ by $w_{a}(j ; \mathbf{q})$ which is defined as

$$
w_{a}(j ; \mathbf{q})=\sqrt{m_{j}} u_{a}(j ; \mathbf{q}) .
$$

$D_{a \beta}^{j k}(\mathbf{q})$ in Equation (8) is called the dynamical matrix, ${ }^{10}$ which takes the form

$$
D_{\alpha \beta}^{j k}(\mathbf{q})=\exp \{i \mathbf{q} \cdot[\mathbf{r}(k)-\mathbf{r}(j)]\}\left[D A_{\alpha \beta}^{j k}(\mathbf{q})+D N_{\alpha \beta}^{j k}(\mathbf{q})\right] .
$$

$D A_{\alpha \beta}^{j k}(\mathbf{q})$ is called the reduced dynamical matrix, ${ }^{10}$ accounting for the analytic contribution under zero-averaged electric field, whereas $D N_{\alpha \beta}^{j k}(\mathbf{q})$ results entirely from the effects of the vibrationinduced macroscopic field. They have the following forms

$$
\begin{aligned}
& D A_{a \beta}^{j k}(\mathbf{q})=\sum_{Q}^{N} \frac{\Phi_{a \beta}^{j k}(P, Q)}{\sqrt{m_{j} m_{k}}} \exp \{i \mathbf{q} \cdot[\mathbf{R}(Q)-\mathbf{R}(P)\} \\
& D N_{a \beta}^{j k}(\mathbf{q})=\frac{4 \pi e[\mathbf{Z}(j) \cdot \hat{\mathbf{q}}]_{\alpha}[\mathbf{Z}(k) \cdot \hat{\mathbf{q}}]_{\beta}}{\sqrt{m_{j} m_{k}} V_{P} \hat{\mathbf{q}} \cdot \boldsymbol{\varepsilon}_{\infty} \cdot \hat{\mathbf{q}}} \frac{1}{N} \sum_{Q}^{N} \exp \{i \mathbf{q} \cdot[\mathbf{R}(Q)-\mathbf{R}(P)]\}
\end{aligned}
$$

The choice of the reference $P$ is arbitrary due to the translational invariance by which $\Phi_{a \beta}^{j k}(P, Q)$ depends on $P$ and $Q$ only through the difference $\mathbf{R}(Q)-\mathbf{R}(P)$. Note that the prefactor term in the right-hand side of Equation (12)

$$
\Delta_{a \beta}^{j k}(\mathbf{q})=\frac{4 \pi e[\mathbf{Z}(j) \cdot \hat{\mathbf{q}}]_{\alpha}[\mathbf{Z}(k) \cdot \hat{\mathbf{q}}]_{\beta}}{\sqrt{m_{j} m_{k}} V_{P} \hat{\mathbf{q}} \cdot \boldsymbol{\varepsilon}_{\infty} \cdot \hat{\mathbf{q}}}
$$

is generally not continuous at $\mathbf{q}=0$ (except for cubic crystals), which is the main reason why contribution given by Equation (12) is called 'nonanalytic'.

With Equation (8), determining phonon frequencies is reduced to finding the eigenvalues for the secular equation

$$
\operatorname{det}\left|D_{a \beta}^{j k}(\mathbf{q})-\omega^{2}(\mathbf{q})\right|=0 .
$$

There are generally $3 N_{p}$ eigenvalues whose roots $\omega_{j}(\mathbf{q})(j=1,2, \ldots$, $3 N_{\mathrm{P}}$ ), are the normal phonon frequencies.

\section{HELMHOLTZ ENERGY AND QUASIHARMONIC APPROXIMATION}

Once the phonon frequencies are obtained, all the thermodynamic quantities can be calculated using statistical physics without further approximations. Neglecting the electron-phonon coupling and the thermal electronic contributions, it is a welldemonstrated procedure ${ }^{5}$ to decompose the Helmholtz energy $F$ of a system at temperature $T$ into two additive contributions as follows

$$
F\left(V_{P}, T\right)=E_{c}(V)+F_{v i b}(V, T),
$$

where $E_{c}$ is the static total energy per primitive unit cell at $0 \mathrm{~K}$, and $F_{\text {vib }}$ is the vibrational contribution to the Helmholtz energy given by ${ }^{46}$

$$
F_{v i b}(V, T)=k_{\mathrm{B}} T \int_{0}^{\infty} \ln \left[2 \sinh \frac{\hbar \omega}{2 k_{B} T}\right] g(\omega, V) d \omega,
$$

where $k_{\mathbf{B}}$ is the Boltzmann constant and $g(\omega, V)$ is the phonon density of states.

The term 'quasiharmonic approximation' arises from the approach that for a given volume, $F_{\mathrm{vib}}(V, T)$ is calculated under the harmonic approximation, and the anharmonic effects are included solely through the volume dependence of the phonon frequency. Once the Helmholtz energy is calculated as a function of volume and temperature, other thermodynamic quantities can be calculated as usual, such as entropy $S=-(\partial F / \partial T)_{V}$, enthalpy $H=F+T S$ and so on.

\section{THE MIXED-SPACE APPROACH}

The procedure presented in the above section represents a combined solution to the phonon problem for polar materials that (i) the long-ranged coulombic interactions are accounted for through Equation (12) in the reciprocal space through $\mathbf{Z}$ and $\boldsymbol{\varepsilon}_{\infty}$ calculated at $\mathbf{q}=0$; and (ii) short-ranged interatomic interactions are accounted for through Equation (11) in terms of $\Phi_{a \beta}^{j k}(P, Q)$ by a supercell in the real space. We therefore refer our solution as mixed-space approach.

In this section, we discuss how the analytic and the nonanalytic contributions are related to the supercell geometry and the type 
of wave-vector points for evaluating the normal vibration frequencies of a polar solid. Let us first examine the nonanalytic contribution to the dynamical matrix in Equation (12) from which we can extract the mathematical geometry factor

$$
f(\mathbf{q})=\frac{1}{N} \sum_{Q}^{N} \exp \{i \mathbf{q} \cdot[\mathbf{R}(Q)-\mathbf{R}(P)]\} .
$$

The values for $f(\mathbf{q})$ depends on whether $\mathbf{q}$ is one of the exact wave-vector points $^{10,16,47}$ in the direct approach. ${ }^{21,37}$ The exact wave-vector points satisfy the following condition

$\mathbf{q}_{e x} \cdot \mathbf{S}_{i}=2 \pi \times$ Integer

where $\mathbf{S}_{i}$ with $i=1,2$, and 3 represent the three lattice vectors of the supercell in the direct approach.

(i) if $\mathbf{q}$ is an exact wave-vector point, i.e., at $\mathbf{q}=\mathbf{q}_{\mathrm{ex}}$ (see Figure 1a). This is the case that no corrections are needed in calculating the dynamical matrix, i.e., the interatomic force constants calculated by the direct approach can be used directly in calculating the dynamical matrix. The internal dipole-dipole interactions within the supercell are already accounted at all exact wave-vector points except for the point at $\mathbf{q}=0$.

(ii) $f(\mathbf{q}) \neq 0$ if $\mathbf{q}$ is not an exact wave-vector point (see Figure $1 b$ ). In this case, phonon properties are determined based on the interatomic force constants obtained with a supercell that is incommensurate with the wavelength corresponding to the wave-vector $\mathbf{q}$. As a result, an artificial electric field is introduced by the incomplete supercell and must be accounted for as a correction to those interatomic force constants calculated under zero macroscopic electric field.

(iii) $f(0)=1$. This is the limiting case as $\mathbf{q} \rightarrow 0$ of case (ii) in which all the local polarizations within the supercell would be equal to each other. The long-wavelength limit $\mathbf{q} \rightarrow 0$ of Equation (12) recovers the results by Cochran and Cowley. ${ }^{34}$

\section{COMPUTATIONAL PROCEDURE}

An actual first-principles phonon calculation of polar solids can be summarized as follows: ${ }^{13,37}$

(i) Calculate the interatomic force constants $\Phi_{\alpha \beta}^{j k}(P, Q)$ in the right-hand side of Equation (11) in the real space, ${ }^{4,48}$ based on a designated supercell; or calculate the dynamical matrix $D_{a \beta}^{j k}(\mathbf{q})$ in Equation (8) in the wave-vector space based on a designated wave-vector grid. ${ }^{16}$

(ii) Calculate the dielectric constant and Born effective charge tensors used in Equation (13) based on the primitive cell by employing either the linear-response approach ${ }^{42}$ or the Berry phase expressions of electric polarization. ${ }^{44}$

(iii) Combine the dielectric properties from step (ii) with $\Phi_{\alpha \beta}^{j k}(P, Q)$ in step (i) to interpolate the phonon frequencies at any wave-vector points. ${ }^{13,37}$

It should be reiterated that in step (i), an implicit condition is that the averaged electric field or the macroscopic electric field is zero. The enforcement of the zero macroscopic electric-field condition is due to the periodic condition adopted in most computer codes for the total electronic energy calculations. For step (iii), a tedious procedure exists in several major computer software packages employing the linear-response approach ${ }^{20,49}$ by separating the dipole-dipole interaction from the short-range interactions. In comparison, except for the codes based on the mixed-space approach, ${ }^{36,37}$ other computational implementations s $^{25-27}$ of step (iii) in the direct approach are inaccurate.

\section{PHONON SOFTWARE PACKAGES HAVE IMPLEMENTED THE MIXED-SPACE APPROACH}

The mixed-space approach has been adopted in a number of software packages, including $\mathrm{YPHON}^{23}$ ShengBTE, ${ }^{8} \mathrm{CRYSTAL}^{28}$ PhonTS, $^{7}$ Phonopy $^{18}$ and ALAMODE. ${ }^{24}$ In these codes, a generalized force constant $\psi_{a \beta}^{j k}(P, Q)$ was introduced as

$$
\psi_{a \beta}^{j k}(P, Q)=\Phi_{a \beta}^{j k}(P, Q)+\phi_{a \beta}^{j k}(\hat{\mathbf{q}}),
$$

where

$$
\phi_{\alpha \beta}^{j k}(\hat{\mathbf{q}})=\frac{1}{N} \frac{4 \pi e[\mathbf{Z}(j) \cdot \hat{\mathbf{q}}]_{\alpha}[\mathbf{Z}(k) \cdot \hat{\mathbf{q}}]_{\beta}}{V_{P} \hat{\mathbf{q}} \cdot \hat{\boldsymbol{\varepsilon}}_{\infty} \cdot \hat{\mathbf{q}}} .
$$

Therefore, the evaluation of the dynamical matrix in Equation (14) becomes

$$
D_{a \beta}^{j k}(\mathbf{q})=\exp \{i \mathbf{q} \cdot[\mathbf{r}(k)-\mathbf{r}(j)]\} \sum_{Q}^{N} \frac{\Psi_{a \beta}^{j k}(P, Q)}{\sqrt{m_{j} m_{k}}} \exp \{i \mathbf{q} \cdot[\mathbf{R}(Q)-\mathbf{R}(P)\} .
$$

This greatly simplifies the computational procedure since one only needs to add a constant term to the calculated force constants by the direct approach. In this respect, the mixed-space approach is a generalization of the approach for the specific case of $\mathrm{GaAs}^{50}$ It should be pointed out that the mixed-space approach is significantly different from previous implementations accounting for the presence of a macroscopic field ${ }^{51}$ in the linear-response approach, ${ }^{14,20}$ where rather tedious and expensive mathematical calculations are involved in order to decompose the calculated interatomic force constants into the short-range contributions and the long-range one from the polar effects.

$\mathrm{YPHON}^{23}$ is an open-source code $(\mathrm{c}++)$ for the calculations of phonon dispersions and phonon density of states.

ShengBTE is a software package for computing the lattice thermal conductivity of crystalline materials and nanowires with diffusive boundary conditions by Li et al. ${ }^{8}$ Both the linear-response approach and the mixed-space approach were implemented in ShengBTE.

The CRYSTAL package performs $a b$ initio calculations of the ground state energy, energy gradient, electronic wave function, and properties of periodic systems. Hartree-Fock or Kohn-Sham Hamiltonians (that adopt an exchange-correlation potential following the postulates of the density functional theory) can be used. Like ShengBTE, PhonTS developed by Chernatynskiy and Phillpot ${ }^{7}$ is a code mainly for thermal conductivity calculations. The mixed-space approach has been implemented in PhonTS exampled by the calculation of phonon lifetime and thermal conductivity of $\mathrm{UO}_{2}$. $^{52,53}$

Phonopy is a Python code developed by Togo ${ }^{18}$ for phonon, thermodynamic properties and thermal conductivity calculations. The mixed-space approach has been implemented in Phonopy for the phonon properties, replacing that by Parliński et al. ${ }^{25-27}$ In particular, a formulation for the evaluation of the third-order force constants is also derived by Togo et al., ${ }^{9}$ extending the mixed-space approach.

ALAMODE (Anharmonic LAttice MODEI) ${ }^{24}$ is designed for estimating harmonic and anharmonic properties of lattice vibrations (phonons) in solids.

\section{RECENT CALCULATIONS USING THE MIXED-SPACE APPROACH}

Phonon and thermodynamic properties

Using the mixed-space approach, phonon and associated properties have been studied for a variety of polar (and non-polar) solids. Most of these calculations are based on the direct approach using the output data from first-principles codes such as VASP ${ }^{11,12}$ as input. Examples are firstly shown for several energy conversion and storage materials. 
Polar crystal $\mathrm{Li}_{2} \mathrm{~S}$ with the anti-fluorite structure and band gap of $4.4 \mathrm{eV}$ is a major compound in Li-S batteries. ${ }^{54}$ Phonons of $\mathrm{Li}_{2} \mathrm{~S}$ have been calculated $^{55}$ using the mixed-space approach. In addition to a nearly perfect agreement between experimental and calculated dispersions of $\mathrm{Li}_{2} \mathrm{~S}$, a large LO-TO splitting was found for the $\mathrm{T}_{1 \mathrm{u}}$ infrared (IR) mode of $\mathrm{Li}_{2} \mathrm{~S} \quad(\sim 30 \%$ and $>120 \mathrm{~cm}^{-1}$ ).

Chalcogenide $\mathrm{Cu}_{2} \mathrm{ZnSn}(\mathrm{S}, \mathrm{Se})_{4}$ (labelled as CZTSSe) with the kesterite structure (space group $P \overline{4} 2 \mathrm{c}$ ) and band gap $1.0 \sim 1.5 \mathrm{eV}$ is a photovoltaic absorber material that has helped achieving significant recent improvement in photovoltaic device cell efficiency $(12.6 \%){ }^{56}$ Phonon frequencies at the $\Gamma$ point and phonon density of state of CZTS were calculated using the mixedspace approach, a 64-atom supercell, and a combined PBEsol ${ }^{57}$ and HSE06. A gap band in the phonon density of state (from $170 \sim 250 \mathrm{~cm}^{-1}$ ) was predicted for CZTS, and especially a negative thermal expansion was suggested at low temperatures (e.g., $<50 \mathrm{~K})$ in terms of phonon density of states.

$\mathrm{LiMPO}_{4}(\mathrm{M}=\mathrm{Mn}, \mathrm{Fe}, \mathrm{Co}$, and $\mathrm{Ni})$ compounds with the olivine structure (space group Pnma) are a class of cathode materials and viable alternatives to the conventional cathode $\mathrm{LiCoO}_{2}{ }^{58} \mathrm{By}$ means of the mixed-space approach, a X-C functional of GGA+U (refs 59,60; $U$ is used to account for the strong on-site Coulomb interaction in transition metals), and a 112-atom supercell, a comparative phonon study has been performed for the antiferromagnetic $\mathrm{LiMPO}_{4}$, and in turn, the associated thermodynamics and bonding strength (the strongest one being $\mathrm{P}-\mathrm{O}$ bonding) have been reported. ${ }^{58}$

$\mathrm{Li}_{2} \mathrm{CO}_{3}$ with space group $\mathrm{C} 2 / \mathrm{c}$ and band gap $5.0 \mathrm{eV}$ has been identified as a main component of the solid electrolyte interphase -a passivating film that forms on Li-ion battery anode surfaces. ${ }^{61}$ Phonons of $\mathrm{Li}_{2} \mathrm{CO}_{3}$ were predicted using the mixed-space approach (including the LO-TO splitting and using a 96-atom supercell).

$\mathrm{TiO}_{2}$ with band gap $~ 3.0 \mathrm{eV}$ has extensive applications such as solar cells, photocatalysts and storage capacitors. Phonon and associated thermodynamics of six $\mathrm{TiO}_{2}$ polymorphs including rutile (space group $\mathrm{P} 4_{2} / \mathrm{mnm}$ ), anatase $\left(14_{1} / \mathrm{amd}\right), \mathrm{TiO}_{2}$-II phase $(\mathrm{Pbcn})$, baddeleyite $\left(\mathrm{P}_{1} / \mathrm{C}\right)$, orthorhombic I (Pbca), and cotunnite (Pnma) were obtained using the mixed-space approach and the $\mathrm{X}-\mathrm{C}$ of the local-density approximation (LDA) ${ }^{62}$ indicating that all $\mathrm{TiO}_{2}$ polymorphs are dynamically stable ${ }^{62}$ and the pressureinduced phase transitions and the pressure-temperature phase diagrams of $\mathrm{TiO}_{2}$ were predicted. ${ }^{63}$

Besides phonons in energy materials, a long-standing issue has been resolved regarding the occurrence of imaginary phonon frequencies in cubic perovskites. These are in fact spurious as they result from the methodology employed. ${ }^{64}$ For example, in perovskites $\mathrm{EuTiO}_{3}$ (ref. 64) and $\mathrm{SrTiO}_{3}{ }^{65}$ a dynamic short-range ordering model using the mixed-space approach as well as the cubic force constants calculated from the low-temperature tetragonal phases was used for phonon calculations. It was seen that the spurious imaginary phonon frequencies in $\mathrm{SrTiO}_{3}$ and $\mathrm{EuTiO}_{3}$ disappear, resulting in a remarkably good agreement with experiments. A similar idea was used for Mott-Hubbard insulators $\mathrm{MnO}$ and $\mathrm{NiO}^{66}$ i.e., the dynamic matrices with the ideal cubic symmetry recovered from the distorted antiferromagnetic structures, the LO-TO splittings estimated using the mixedspace approach, and the strong electron correlations accounted for by the GGA+U method, ${ }^{59}$ which produced accurate phonon dispersions for $\mathrm{MnO}$ and $\mathrm{NiO}$. For room temperature multiferroic $\mathrm{BiFeO}_{3}{ }^{67}$ the challenge in calculating its phonon properties is due to the fact that $\mathrm{BiFeO}_{3}$ is a Mott-Hubbard insulator with band gap $\sim 2.5 \mathrm{eV}$, involving a polar effect and strong correlation among the $d$ electrons of Fe. The mixed-space approach together with the $\mathrm{GGA}+U$ method $^{60}$ accurately predicted the phonon dispersions of $\mathrm{BiFeO}_{3}$ and suggested that no gapped magnon modes ${ }^{68}$ exist and contribute to the heat capacity of $\mathrm{BiFeO}_{3}$ in the temperature range $5-30 \mathrm{~K}$.

Accurate phonon properties have also been predicted for $\mathrm{CaF}_{2}$ and $\mathrm{CeO}_{2}$ with the fluorite structure. ${ }^{69} \mathrm{CaF}_{2}$ is a typical superionic conductor, its phonons have been studied by the PBE exchangecorrelation functional ${ }^{70}$ using a 192-atom supercell. $\mathrm{CeO}_{2}$ has been used in catalytic converters in automotive applications and as an electrolyte in fuel cells because of its relatively high oxygen ion conductivity. In particular for considering the $f$-electron system, phonon dispersions of $\mathrm{CeO}_{2}$ have been studied by a HSE06 hybrid functional, ${ }^{71,72}$ showing better accuracy ${ }^{69}$ than the previous predictions from e.g., PWSCF $^{73}$ and ABINIT. ${ }^{74}$

In addition to the prototype $\mathrm{a}-\mathrm{Al}_{2} \mathrm{O}_{3},{ }^{37}$ phonon and associated thermodynamics were successfully predicted using $L D A+U$ and the mixed-space approach for another dense and continuous coating material of $\mathrm{Cr}_{2} \mathrm{O}_{3}{ }^{75}$ Furthermore, phonon-related properties have been investigated using the mixed-space approach for many other polar solids such as nanograined half-Heusler semiconductors, ${ }^{76}$ calcium fluoride at high pressure, ${ }^{32}$ the phase diagram of bismuth ferrite, ${ }^{33}$ cubic $\mathrm{SiC}$ and hexagonal $\mathrm{BN}^{37} \mathrm{ZnO}^{77}$ $\mathrm{UN}^{78,79} \mathrm{Bi}_{2} \mathrm{~S}_{3}{ }^{80} \mathrm{Si}, \mathrm{Ge}$, InAs and $\mathrm{GaAs}_{1}{ }^{38} \mathrm{GaN}^{81} \mathrm{CrN}^{82} \mathrm{WS}_{2}{ }^{83}$ $\mathrm{ZnSe}^{84} \mathrm{ZnS}, \mathrm{ZnSe}$ and $\mathrm{ZnTe}^{85} \mathrm{SnO}_{2},{ }^{86} \mathrm{TaO}_{3}{ }^{87} \mathrm{UO}_{2}{ }^{52,53,88}$ $\mathrm{CaCO}_{3},{ }^{89} \mathrm{Bi}_{2} \mathrm{SiO}_{5}$ (ref. 90) and $\mathrm{BaZrO}_{3} .{ }^{91}$ layered antimony telluride, ${ }^{92}$ Phonon transport in $\mathrm{SrTiO}_{3}{ }^{93}$ self-consistent phonon calculations for cubic $\mathrm{SrTiO}_{3}$ (ref. 94 ) and $\mathrm{Si}\left(\mathrm{Se}_{\mathrm{x}} \mathrm{S}_{1-x}\right)_{2} \cdot{ }^{95}$

\section{Thermal conductivities of polar solids}

The main heat carriers in nonmagnetic crystals are phonons and electrons, with phonons dominating in semiconductors and insulators. The phonon contribution to the total thermal conductivity is the lattice thermal conductivity. One important approach to studying phonon transport in solids is the Boltzmann transport equation (BTE). ${ }^{96}$ However, many solutions of BTE rely on the relaxation time approximation along with the Debye approximation, neglecting the true phonon dispersions, and several parameters are introduced to treat different scattering mechanisms. Li et $a l^{8}{ }^{8}$ and Chernatynskiy et al. $^{7}$ implemented parameter-free iterative solutions in their software packages (ShengBTE ${ }^{8}$ and PhonTS $^{7}$ ) to solve the BTE based on the inputs from firs principles. The programs compute converged sets of phonon scattering rates and use them to obtain the lattice thermal conductivity and many related quantities. The two main inputs needed by their software packages are sets of second-order (harmonic) and third-order (anharmonic) interatomic force constants (IFCs) for a given crystal structure. Our mixed-space approach has been implemented in both software packages to derive the second-order IFCs, so as to account for the long-range electrostatic interactions in polar compounds. To date, $a b$ initio calculations of lattice thermal conductivity have been applied by $\mathrm{Li}$ et al. for many bulk systems such as $\mathrm{Mg}_{2} \mathrm{Si}, \mathrm{Mg}_{2} \mathrm{Sn}$ and $\mathrm{Mg}_{2} \mathrm{Si}_{x} \mathrm{Sn}_{1-x,}{ }^{97}$ two-dimensional systems such as $\mathrm{MoS}_{2}$ (ref. 98) and nanowires made of $\mathrm{Si}$, diamond, ${ }^{99} \operatorname{InAs}$, AIN and $\mathrm{BeO}^{100}$ under the diffusive boundary conditions. All these applications show excellent agreement with experimental measurements, and an accurate description of polar-polar interactions was found crucial for theoretical predictions to be in line with experiments. As claimed by $\mathrm{Li}$ et al., ${ }^{8,100}$ in their work for $\ln A$ s of a well-known direct-band gap III-V semiconductor, 'Therefore it is ideally well suited for validating our approach when isotope scattering and polar bonds are introduced into the picture'. More examples of lattice thermal conductivity calculations have been reported for $\mathrm{SnSe}^{101} \mathrm{MgO}, \mathrm{GaAs}, \mathrm{SiC}, \mathrm{BN}, \mathrm{BP}, \mathrm{BSb}, \mathrm{BAs}, \mathrm{BeTe}$, and BeSe, ${ }^{102}$ InN ${ }^{103}$ phosphorene ${ }^{104,105}$ and $\mathrm{Si}, \mathrm{Ge}$, InAs and GaAs alloys. ${ }^{106}$ 


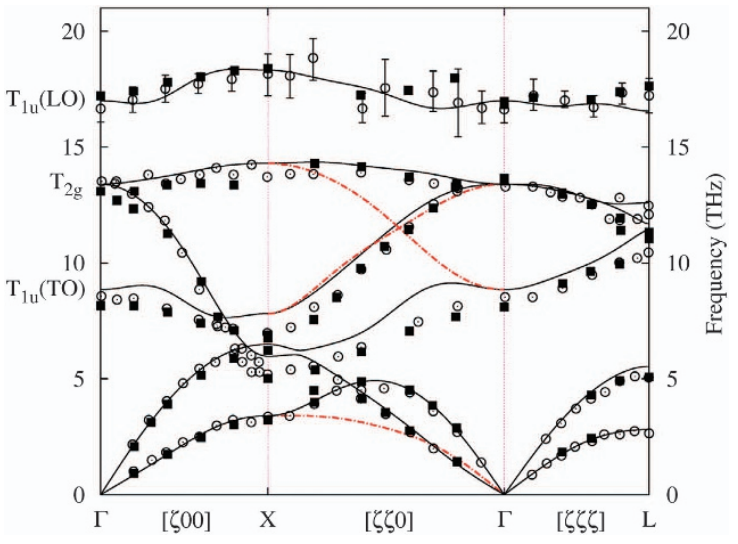

Figure 2. Phonon dispersions of $\mathrm{UO}_{2}$. Open circles: measured data by Pang et al. ${ }^{52}$ solid squares: measured data by Dolling et $a l^{10}{ }^{10}$ The lines represent the present calculations. The (red) dot-dashed lines

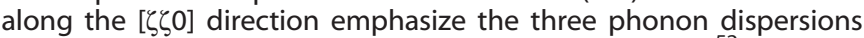
that were not reported by the calculation of Pang et al. ${ }^{52}$

Phonon dispersions for systems with symmetry broken by magnetism

There are systems such as $\mathrm{MnO}, \mathrm{NiO}$, and $\mathrm{UO}_{2}$ (refs 53,66) for which the high-temperature phase is paramagnetic, and the low temperature phase is antiferromagnetic. For these systems, it is too expensive to calculate the phonons of the high-temperature phase accurately. An alternative approximation to calculate the phonons of the high-temperature phase is to use the force constants calculated from the corresponding low-temperature phase. In doing so, one primary problem to solve is the symmetry broken by the magnetic degree of freedom. Using $\mathrm{UO}_{2}$ as an example, the primitive cell of the antiferromagnetic structure contains 6 atoms resulting in 18 phonon dispersions, whereas the primitive cell of the paramagnetic structure contains 3 atoms resulting in 9 phonon dispersions. A solution to the problem is to restore the symmetry by a transformation as

$$
\Phi_{s t}^{\alpha \beta} \text { (high symmetry) }=\frac{1}{S} \sum_{r=1}^{N} \mathbf{O}_{r}^{-1} \Phi_{s t}^{\alpha \beta} \text { (low symmetry) } \mathbf{O}_{r}
$$

where $\Phi_{s t}^{\alpha \beta}$ (high symmetry) represents the force constant matrix of the high-symmetry structure, $\Phi_{s t}^{\alpha \beta}$ (low symmetry) the force constant matrix calculated from the low symmetry structure, $\mathbf{O}_{r}$ the space group operation of the high-symmetry structure, and $S$ the number of $\mathbf{O}_{r}$ 's. Figure 2 shows the phonon dispersions of $\mathrm{UO}_{2}$. It is observed that our calculated phonon dispersions show great improvements over the previous calculations ${ }^{52,107-109}$ by comparing them with the experimental data. ${ }^{52,110}$

\section{OTHER PHONON SOFTWARE PACKAGES}

PHON is an open-source code developed by Alfè ${ }^{21}$ to calculate phonon frequencies following the direct approach by Parliński et $a l^{17}$ ATAT is a generic name that refers to a collection of open-source alloy theory tools developed by van de Walle et al. ${ }^{4,22}$ For phonon calculations, it appears that neither codes can yet handle the vibration-induced polarization effects.

PHONON is a commercial code for phonon and thermal properties developed by Parliński et al. ${ }^{17,25-27}$ As an example, Figure 3 illustrates the phonon dispersions of $a-\mathrm{Al}_{2} \mathrm{O}_{3}$ calculated using the PHONON package by Lodziana and Parliński ${ }^{111}$ in comparison with those calculated using $\mathrm{YPHON}^{37}$ and measured from the inelastic neutron scattering by Schober et al. ${ }^{112}$ It can be seen that the application of PHONON code for phonon dispersion calculations of polar solids appears inaccurate due to an artificial implementation of the Gaussian smear extrapolation ${ }^{25-27}$
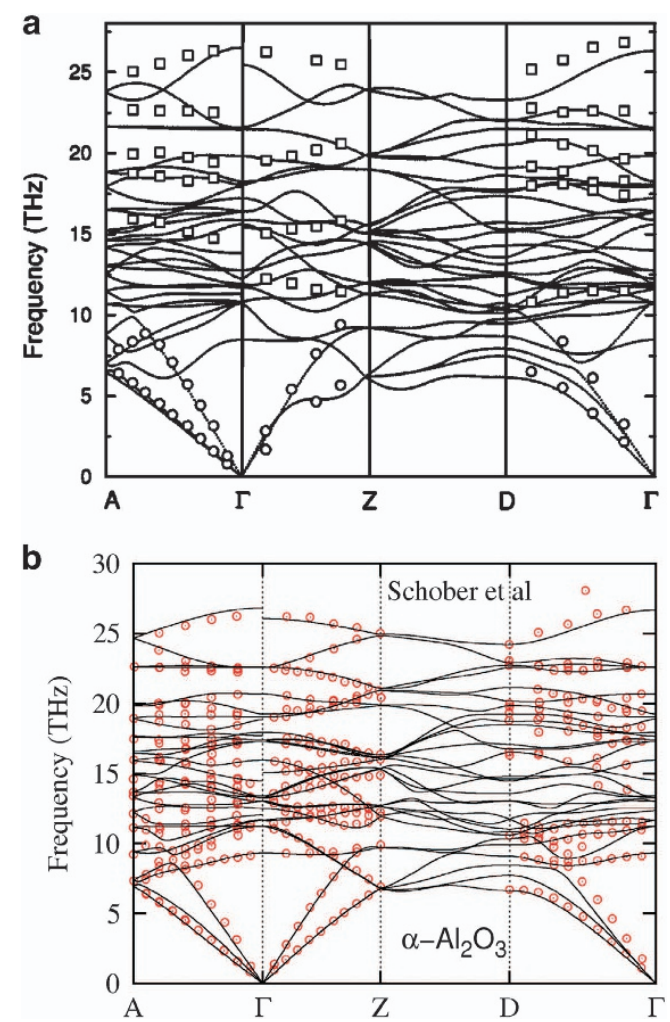

Figure 3. Phonon dispersions for $\mathrm{Al}_{2} \mathrm{O}_{3}$. (a) Calculated using PHONON by Lodziana and Parliński. ${ }^{111}$ (b) Calculated using YPHON by Wang et al. $^{37}$ The symbols represent the inelastic neutron scattering data by Schober et al. ${ }^{112}$

in accounting for the vibration-induced polarization effects. Practically, PHONON code was applied to many polar solids including, for example, $\mathrm{ZrO}_{2}{ }^{17}$ alkaline-earth metals and their hydrides, ${ }^{113} \mathrm{LiBeH}_{3},{ }^{114} \mathrm{CoO}_{1}{ }^{115} \mathrm{Fe}_{2} \mathrm{SiO}_{4}$ spinel, ${ }^{116} \mathrm{Li}_{2} \mathrm{O}$ and $\mathrm{Li}_{2} \mathrm{CO}_{3}{ }^{117} \mathrm{LiF}^{118} \mathrm{CsCl}$ and $\mathrm{BaCl}^{119}$ hafnia and zirconia, ${ }^{120} \mathrm{PuO}_{2}{ }^{121}$ $\mathrm{CeO}_{2}, \mathrm{ThO}_{2}$ and (Ce,Th) $\mathrm{O}_{2}$ alloys, ${ }^{122,123} \mathrm{LiFePO}_{4}{ }^{124} \mathrm{MnO}^{125}$ $\mathrm{ZnO}^{126} \mathrm{BeO}^{127}$ and $\mathrm{BiFeO}_{3}$. ${ }^{128}$

\section{SOFTWARE PACKAGES FOR BOTH ELECTRONIC AND PHONON CALCULATIONS}

The codes widely in use include the open-source packages PWSCF/QUANTUM ESPRESSO ${ }^{14}$ and ABINIT, $^{15}$ and the commercial packages CASTEP, ${ }^{13}$ CRYSTAL $^{28}$ and VASP. ${ }^{11,12}$ For phonon calculations of polar materials, PWSCF, QUANTUM ESPRESSO, ABINIT and CASTEP employ the linear-response approach. If one wants to calculate phonons of a polar solid using CRYSTAL (starting from CRYSTAL $14^{28}$ ), then the mixed-space approach is the only choice.

VASP only calculates the phonon frequencies at the exact wavevector points. Although VASP can calculate the dielectric constant and Born effective charge tensors, the current version of VASP (version 5.4.1.05 Feb16) does not report the LO-TO splitting. ${ }^{34,40}$

PWSCF is one of the core packages of open-source QUANTUM ESPRESSO.$^{14}$ We employed PWSCF to perform the linear-response calculations of phonon properties of $\mathrm{Ni}, \mathrm{Al}, \mathrm{NiAl}$ and $\mathrm{Ni}_{3} \mathrm{Al}_{1}^{5}$ and MgO. ${ }^{129}$ Linear-response approach is also employed for to phonon calculations by $\mathrm{ABINIT}^{15}$ which is an open-source package using pseudopotentials and a planewave basis. The linear-response approach relies on the availability of pseudopotentials in specific formats. For example, the CASTEP ${ }^{13}$ code requires the use of the Norm-conserving pseudopotentials. ${ }^{130}$ To account for the polar effects on phonon calculations, these linear-response codes 
commonly use the following computational procedure ${ }^{14,19,130}$ for the evaluation of the dynamical matrix at an arbitrary $\mathbf{q}$ points:

(i) Calculate the dynamical matrices at a predetermined reference coarse grid

(ii) Remove the long-ranged coulombic contribution from the dynamical matrices that are calculated at the coarse wave-vector grid

(iii) Make backward Fourier-transform of the dynamical matrices to obtain the interatomic interaction constants and

(iv) For an arbitrary $\mathbf{q}$ point outside the coarse wave-vector grid, make forward Fourier-transform of the interatomic interaction constants followed by re-adding the longranged coulombic contribution to get the dynamical matrix at the arbitrary q points.

These steps were based on the belief from previous conclusions $^{14,19}$ that for solids having TO-LO splitting (e.g.: polar semiconductors), as the force constants in real space involves long-ranged interatomic interactions due to the nonanalytic term, Fourier interpolation is no longer possible. In contrast to this belief, we note that by using the mixed-space approach, the Fourier interpolation is possible and computationally efficient. One example was given by Zhao et $a l^{80}$ in their phonon calculations of $\mathrm{Bi}_{2} \mathrm{~S}_{3}$ nanostructures using QUANTUM ESPRESSO. Instead of using the linear-response approach as implemented in QUANTUM ESPRESSO, Zhao et al. ${ }^{80}$ employed the mixed-space approach and they found the mixed-space approach is more efficient without sacrificing the accuracy.

\section{SUMMARY}

This paper reviews a mixed-space formulation to treat the contribution of vibration-induced polarization to phonon dispersions in the direct or supercell approach. It decomposes the interatomic force constants into two contributions: one for all internal interactions within the supercell under zero macroscopic electric field and the other one accounts for the effects of nonzero macroscopic electric field arising from supercells that are incommensurate with the wavelengths of the lattice vibrations. The theory naturally gives rise to the analytic and the nonanalytic contributions to the total force constants at any wave-vector points, without the assumption of the long-wavelength limit made by Cochran and Cowley. ${ }^{34}$ It provides a useful methodology separating the dipole-dipole interaction from those short-range interactions for Fourier interpolation. ${ }^{16,20,49}$ It has been successfully applied in calculating the phonon and thermal properties of a wide range of polar materials and implemented in several broadly used software packages for calculating phonon properties.

\section{ACKNOWLEDGEMENTS}

This work was supported by the U.S. Department of Energy, Office of Basic Energy Sciences, Division of Materials Sciences and Engineering under Award DE-FG02-07ER46417 (Wang and Chen) and by National Science Foundation (NSF) through Grant Nos. DMR-1310289 and CHE-1230924 (Wang, Shang, Fang, and Liu). First-principles calculations were carried out partially on the LION clusters at the Pennsylvania State University, partially on the resources of NERSC supported by the Office of Science of the U.S. Department of Energy under contract No. DE-AC02-05CH11231, and partially on the resources of XSEDE supported by NSF with Grant No. ACl-1053575.

\section{COMPETING INTERESTS}

The authors declare no conflict of interest.

\section{REFERENCES}

1. Tamm, I. Über die quantentheorie der molekularen lichtzerstreuung in festen körpern. Z. Phys. 60, 345-363 (1930).

2. Born, M. \& Huang, K. Dynamical Theory of Crystal Lattices (Clarendn, Oxford, 1954).

3. Mittal, R., Chaplot, S. L. \& Choudhury, N. Modeling of anomalous thermodynamic properties using lattice dynamics and inelastic neutron scattering. Prog. Mater. Sci. 51, 211-286 (2006).

4. van de Walle, A., Asta, M. \& Ceder, G. The alloy theoretic automated toolkit: a user guide. Calphad 26, 539-553 (2002).

5. Wang, Y., Liu, Z. K. \& Chen, L. Q. Thermodynamic properties of Al, Ni, NiAl, and Ni3Al from first-principles calculations. Acta Mater. 52, 2665-2671 (2004).

6. Wang, Y. et al. A first-principles approach to finite temperature elastic constants. J. Phys. Cond. Mat. 22, 225404 (2010).

7. Chernatynskiy, A. \& Phillpot, S. R. Phonon transport simulator (PhonTS). Comput. Phys. Commun. 192, 196-204 (2015).

8. Li, W., Carrete, J., Katcho, N. A. \& Mingo, N. ShengBTE: a solver of the Boltzmann transport equation for phonons. Comput. Phys. Commun. 185, 1747-1758 (2014).

9. Togo, A., Chaput, L. \& Tanaka, I. Distributions of phonon lifetimes in Brillouin zones. Phys. Rev. B 91, 094306 (2015).

10. Wallace, D. C. Thermodynamics of Crystals (Joha Wiley \& Sons, Inc., 1972).

11. Kresse, G. \& Furthmuller, J. Efficiency of ab-initio total energy calculations for metals and semiconductors using a plane-wave basis set. Comput. Mater. Sci. 6, 15-50 (1996).

12. Kresse, G. \& Joubert, D. From ultrasoft pseudopotentials to the projector augmented-wave method. Phys. Rev. B 59, 1758-1775 (1999).

13. Clark, S. J. et al. First principles methods using CASTEP. Z. Kristallogr. 220, 567-570 (2005).

14. Giannozzi, P. et al. QUANTUM ESPRESSO: a modular and open-source software project for quantum simulations of materials. J. Phys. Condens. Matter 21, 395502 (2009).

15. Gonze, X. et al. ABINIT: first-principles approach to material and nanosystem properties. Comput. Phys. Commun. 180, 2582-2615 (2009).

16. Baroni, S., de Gironcoli, S., Dal Corso, A. \& Giannozzi, P. Phonons and related crystal properties from density-functional perturbation theory. Rev. Mod. Phys. 73, 515-562 (2001).

17. Parliński, K., Li, Z. Q. \& Kawazoe, Y. First-principles determination of the soft mode in cubic ZrO2. Phys. Rev. Lett. 78, 4063-4066 (1997).

18. Togo, A., Oba, F. \& Tanaka, I. First-principles calculations of the ferroelastic transition between rutile-type and $\mathrm{CaCl} \_\{2\}$-type $\mathrm{SiO} \_\{2\}$ at high pressures. Phys. Rev. B 78, 134106 (2008).

19. Giannozzi, P., Baroni, S. in Handbook of Materials Modeling (ed. Yip S.) 195-214 (Springer Science \& Business Media, 2007).

20. Gonze, X. \& Lee, C. Dynamical matrices, born effective charges, dielectric permittivity tensors, and interatomic force constants from density-functional perturbation theory. Phys. Rev. B 55, 10355-10368 (1997).

21. Alfè, D. PHON: a program to calculate phonons using the small displacement method. Comput. Phys. Commun. 180, 2622-2633 (2009).

22. van de Walle, A. \& Ceder, G. The effect of lattice vibrations on substitutional alloy thermodynamics. Rev. Mod. Phys. 74, 11-45 (2002).

23. Wang, Y., Chen, L.-Q. \& Liu, Z.-K. YPHON: a package for calculating phonons of polar materials. Comput. Phys. Commun. 185, 2950-2968 (2014).

24. Tadano, T. ALAMODE Documentation Release 0.9.7. Available at https://media. readthedocs.org/pdf/alamode/latest/alamode.pdf (2016).

25. Adeagbo, W. A. \& Entel, P. Influence of dipole interactions on the lattice dynamics of crystalline ice. Phase Transitions 78, 799-810 (2005).

26. Parliński, K., Lazewski, J. \& Kawazoe, Y. Ab initio studies of phonons in MgO by the direct method including LO mode. J. Phys. Chem. Solids 61, 87-90 (2000).

27. Parliński, K., Li, Z. Q. \& Kawazoe, Y. Long-range Coulomb interaction in $\mathrm{ZrO} 2$ (reply to Parlinski, K., Z. Q. Li, and Y. Kawazoe). Phys. Rev. Lett. 81, 3298-3298 (1998).

28. Dovesi, R. et al. CRYSTAL14: A program for the $a b$ initio investigation of crystalline solids. Int. J. Quantum Chem. 114, 1287-1317 (2014).

29. Ye, Z.-Y. et al. The origin of electronic band structure anomaly in topological crystalline insulator group-IV tellurides. npj Comput. Mater. 1, 15001 (2015).

30. Scott, J. F. Searching for new ferroelectrics and multiferroics: a user's point of view. npj Comput. Mater 1, 15006 (2015).

31. Alharbi, F. H. et al. An efficient descriptor model for designing materials for solar cells. npj Comput. Mater 1, 15003 (2015).

32. Cazorla, C. \& Errandonea, D. Superionicity and Polymorphism in calcium fluoride at high pressure. Phys. Rev. Lett. 113, 235902 (2014).

33. Cazorla, C. \& Iniguez, J. Insights into the phase diagram of bismuth ferrite from quasiharmonic free-energy calculations. Phys. Rev. B 88, 214430 (2013). 
34. Cochran, W. \& Cowley, R. A. Dielectric constants and lattice vibrations. J. Phys. Chem. Solids 23, 447-450 (1962).

35. Tulip, P. R. Dielectric and lattice dynamical properties of molecular crystals via density functional perturbation theory: implementation within a first principles code (PhD Thesis, Durham University, 2004).

36. Wang, Y., Shang, S. L., Liu, Z. K. \& Chen, L. Q. Mixed-space approach for calculation of vibration-induced dipole-dipole interactions. Phys. Rev. B 85, 224303 (2012)

37. Wang, Y. et al. A mixed-space approach to first-principles calculations of phonon frequencies for polar materials. J. Phys. Condes. Matter 22, 202201 (2010).

38. Vermeersch, B., Carrete, J., Mingo, N. \& Shakouri, A. Superdiffusive heat conduction in semiconductor alloys. I. Theoretical foundations. Phys. Rev. B 91, 085202 (2015).

39. Herman, F. Lattice vibrational spectrum of germanium. J. Phys. Chem. Solids 8 405-418 (1959)

40. Kunc, K. \& Martin, R. M. Ab initio force constants of GaAs: a new approach to calculation of phonons and dielectric properties. Phys. Rev. Lett. 48, 406-409 (1982).

41. Churchill, R. V., Brown, J. W. \& Verhey, R. F. Complex Variables and Applications (McGraw-Hill, 1974).

42. Gajdoš, M., Hummer, K., Kresse, G., Furthmuller, J. \& Bechstedt, F. Linear optical properties in the projector-augmented wave methodology. Phys. Rev. B 73, 045112 (2006).

43. Durman, R., Favre, P., Jayasooriya, U. A. \& Kettle, S. F. A. Longitudinal optical transverse optical (LO-TO) splitting on internal-modes in the RAMAN-spectra of noncentric crystals. J. Crystallogr. Spectrosc. Res. 17, 431-484 (1987).

44. Resta, R. Macroscopic polarization in crystalline dielectrics: the geometric phase approach. Rev. Mod. Phys. 66, 899-915 (1994).

45. Kittel, C. Introduction to solid state physics 8th edn (Wiley, Hoboken, NJ, 2005).

46. Xie, J. J., de Gironcoli, S., Baroni, S. \& Scheffler, M. First-principles calculation of the thermal properties of silver. Phys. Rev. B 59, 965-969 (1999).

47. Kern, G., Kresse, G. \& Hafner, J. Ab initio calculation of the lattice dynamics and phase diagram of boron nitride. Phys. Rev. B 59, 8551-8559 (1999).

48. Parliński, K. Phonon Software, MedeA 1.8. Materials Design (2007).

49. Giannozzi, P., de Gironcoli, S., Pavone, P. \& Baroni, S. Ab initio calculation of phonon dispersions in semiconductors. Phys. Rev. B 43, 7231-7242 (1991).

50. Kunc, K. \& Martin, R. M. Ab initio force-constants of GaAS-a new approach to calculation of phonons and dielectric-properties. Phys. Rev. Lett. 48, 406-409 (1982).

51. Pick, R. M., Cohen, M. H. \& Martin, R. M. Microscopic theory of force constants in adiabatic approximation. Phys. Rev. B 1, 910-920 (1970).

52. Pang, J. W. L. et al. Phonon lifetime investigation of anharmonicity and thermal conductivity of UO2 by neutron scattering and theory. Phys. Rev. Lett. 110, 157401 (2013).

53. Pang, J. W. L. et al. Phonon density of states and anharmonicity of UO2. Phys. Rev. B 89, 115132 (2014).

54. Shang, S. et al. Insight into structural, elastic, phonon, and thermodynamic properties of alpha-sulfur and energy-related sulfides: a comprehensive firstprinciples study. J. Mater. Chem. A 3, 8002-8014 (2015).

55. Shang, S., Wang, Y. \& Liu, Z.-K. First-principles thermodynamics at finite temperatures: Perspective on ordered and disordered phases. Mater. China 34, 297-304 (2015).

56. Shang, S. et al. Cation disorder regulation by microstate configurational entropy in photovoltaic absorber materials Cu2ZnSn(S,Se)(4). J. Phys. Chem. C 118 24884-24889 (2014).

57. Paier, J. et al. Screened hybrid density functionals applied to solids. J. Chem Phys. 124, 154709 (2006).

58. Shang, S. L., Wang, Y., Mei, Z. G., Hui, X. D. \& Liu, Z. K. Lattice dynamics, thermodynamics, and bonding strength of lithium-ion battery materials LiMPO4 ( $\mathrm{M}=\mathrm{Mn}, \mathrm{Fe}, \mathrm{Co}$, and Ni): a comparative first-principles study. J. Mater. Chem. 22, 1142-1149 (2012).

59. Dudarev, S. L., Botton, G. A., Savrasov, S. Y., Humphreys, C. J. \& Sutton, A. P. Electron-energy-loss spectra and the structural stability of nickel oxide: An LSDA + U study. Phys. Rev. B 57, 1505-1509 (1998).

60. Liechtenstein, A. I., Anisimov, V. I. \& Zaanen, J. Density-functional theory and strong-interactions-orbital ordering in Mott-Hubbard insulators. Phys. Rev. B 52, R5467-R5470 (1995).

61. Shang, S. L. et al. Lattice dynamics, thermodynamics and elastic properties of monoclinic $\mathrm{Li} 2 \mathrm{CO} 3$ from density functional theory. Acta Mater. 60 , 5204-5216 (2012)

62. Mei, Z. G., Wang, Y., Shang, S. L. \& Liu, Z. K. First-principles study of the lattice dynamics and thermodynamics of $\mathrm{TiO} 2$ polymorphs. Inorg. Chem. 50, 6996-7003 (2011)

63. Mei, Z.-G., Wang, Y., Shang, S. \& Liu, Z.-K. First-principles study of the mechanical properties and phase stability of TiO2. Comput. Mater. Sci. 83, 114-119 (2014).
64. Wang, Y., Shang, S., Chen, L.-Q. \& Liu, Z.-K. Density functional theory-based database development and CALPHAD automation. JOM 65, 1533-1539 (2013).

65. Wang, Y. et al. A first-principles scheme to phonons of high temperature phase: no imaginary modes for cubic SrTiO3. Appl. Phys. Lett. 97, 162907 (2010).

66. Wang, Y. et al. Broken symmetry, strong correlation, and splitting between longitudinal and transverse optical phonons of $\mathrm{MnO}$ and $\mathrm{NiO}$ from first principles. Phys. Rev. B 82, 081104 (2010).

67. Wang, Y. et al. First-principles lattice dynamics and heat capacity of BiFeO3. Acto Mater. 59, 4229-4234 (2011).

68. Lu, J. et al. On the room temperature multiferroic BiFeO3: magnetic, dielectric and thermal properties. Eur. Phys. J. B 75, 451-460 (2010).

69. Wang, Y., Zhang, L. A., Shang, S., Liu, Z.-K. \& Chen, L.-Q. Accurate calculations of phonon dispersion in CaF2 and CeO2. Phys. Rev. B 88, 024304 (2013).

70. Perdew, J. P., Burke, K. \& Ernzerhof, M. Generalized gradient approximation made simple. Phys. Rev. Lett. 77, 3865-3868 (1996).

71. Heyd, J., Scuseria, G. E. \& Ernzerhof, M. Hybrid functionals based on a screened Coulomb potential. J. Chem. Phys. 118, 8207-8215 (2003).

72. Heyd, J., Scuseria, G. E. \& Ernzerhof, M. Hybrid functionals based on a screened Coulomb potential. 118, 8207 (2003) J. Chem. Phys (Erratum in Hybrid functionals based on a screened Coulomb potential, J. Chem. Phys. 124, 219906 (2006).

73. Verstraete, M. \& Gonze, X. First-principles calculation of the electronic, dielectric and dynamical properties of CaF2. Phys. Rev. B 68, 195123 (2003).

74. Gürel, T. \& Eryiğit, R. Ab initio pressure-dependent vibrational and dielectric properties of CeO2. Phys. Rev. B 74, 014302 (2006).

75. Wang, Y. et al. First-principles lattice dynamics, thermodynamics, and elasticity of Cr2O3. Surf. Sci. 606, 1422-1425 (2012).

76. Carrete, J., Mingo, N., Wang, S. \& Curtarolo, S. Nanograined half-Heusler semiconductors as advanced thermoelectrics: an ab initio high-throughput statistical study. Adv. Eng. Mater. 24, 7427-7432 (2014).

77. Calzolari, A. \& Nardelli, M. B. Dielectric properties and Raman spectra of $\mathrm{ZnO}$ from a first principles finite-differences/finite-fields approach. Sci. Rep. 3, 2999 (2013).

78. Mei, Z.-G. \& Stan, M. Pressure-induced phase transitions in UN: a density functional theory study. J. Alloys Compd 588, 648-653 (2014).

79. Mei, Z.-G., Stan, M. \& Pichler, B. First-principles study of structural, elastic, electronic, vibrational and thermodynamic properties of UN. J. Nucl. Mater. 440, 63-69 (2013).

80. Zhao, Y. et al. Phonons in Bi2S3 nanostructures: Raman scattering and firstprinciples studies. Phys. Rev. B 84, 205330 (2011).

81. Zhou, Y., Wang, S., Wang, R. \& Jiang, N. Ab initio calculation of the thermodynamic properties and phase diagram of gallium nitride. Physica $B$ 431, 115-119 (2013).

82. Zhou, L. et al. Structural stability and thermodynamics of $\mathrm{CrN}$ magnetic phases from ab initio calculations and experiment. Phys. Rev. B 90, 184102 (2014).

83. Gandi, A. N. \& Schwingenschloegl, U. WS2 as an excellent high-temperature thermoelectric material. Chem. Mater. 26, 6628-6637 (2014).

84. Zhang, X. et al. Zincblende-wurtzite phase transformation of ZnSe films by pulsed laser deposition with nitrogen doping. Appl. Phys. Lett. 103, 082111 (2013).

85. Katre, A., Togo, A., Tanaka, I. \& Madsen, G. K. H. First principles study of thermal conductivity cross-over in nanostructured zinc-chalcogenides. J. Appl. Phys. 117, 045102 (2015)

86. Dou, M. \& Persson, C. Comparative study of rutile and anatase $\mathrm{SnO} 2$ and $\mathrm{TiO} 2$ : Band-edge structures, dielectric functions, and polaron effects. J. Appl. Phys. 113, 083703 (2013).

87. Ravi, C., Kaur, G. \& Bharathi, A. First-principles study of lattice stability of ReO3type hypothetical TaO3. Comput. Mater. Sci. 90, 177-181 (2014).

88. Mei, Z.-G., Stan, M. \& Yang, J. First-principles study of thermophysical properties of uranium dioxide. J. Alloys Compd. 603, 282-286 (2014).

89. Zhang, Z. \& Liu, Z. High pressure equation of state for molten $\mathrm{CaCO} 3$ from first principles simulations. Chinese J. Geochem. 34, 13-20 (2015).

90. Taniguchi, H. et al. Ferroelectricity driven by twisting of silicate tetrahedral chains. Angew. Chem., Int. Ed. 52, 8088-8092 (2013).

91. Bjørheim, T. S., Kotomin, E. A. \& Maier, J. Hydration entropy of BaZrO 3 from first principles phonon calculations. J. Mater. Chem. A 3, 7639-7648 (2015).

92. Stoffel, R. P., Deringer, V. L., Simon, R. E., Hermann, R. P. \& Dronskowski, R. A density-functional study on the electronic and vibrational properties of layered antimony telluride. J. Phys.: Condens. Matter 27, 085402 (2015).

93. Feng, L., Shiga, T. \& Shiomi, J. Phonon transport in perovskite SrTiO3 from first principles. Appl. Phys. Express 8, 071501 (2015).

94. Tadano, T. \& Tsuneyuki, S. Self-consistent phonon calculations of lattice dynamical properties in cubic $\mathrm{SrTiO} 3$ with first-principles anharmonic force constants. Phys. Rev. B 92, 054301 (2015) 
95. Chen, C. et al. Synthesis, characterization and chemical stability of silicon dichalcogenides, Si (Se x S 1-x) 2. J. Cryst. Growth. doi:10.1016/j.jcrysgro.2015. 12.005 (2016).

96. Ziman, J. M. Electrons and Phonons: The Theory of Transport Phenomena in Solids (Clarendon Press, 1960).

97. Li, W., Lindsay, L., Broido, D. A., Stewart, D. A. \& Mingo, N. Thermal conductivity of bulk and nanowire Mg2SixSn1-x alloys from first principles. Phys. Rev. B 86, 174307 (2012).

98. Li, W., Carrete, J. \& Mingo, N. Thermal conductivity and phonon linewidths of monolayer MoS2 from first principles. Appl. Phys. Lett. 103, 253103 (2013).

99. Li, W. et al. Thermal conductivity of diamond nanowires from first principles. Phys. Rev. B 85, 195436 (2012).

100. Li, W. \& Mingo, N. Thermal conductivity of bulk and nanowire InAs, AIN, and BeO polymorphs from first principles. J. Appl. Phys. 114, 183505 (2013).

101. Carrete, J., Mingo, N. \& Curtarolo, S. Low thermal conductivity and triaxial phononic anisotropy of SnSe. Appl. Phys. Lett. 105, 101907 (2014).

102. Lindsay, L., Broido, D. A., Carrete, J., Mingo, N. \& Reinecke, T. L. Anomalous pressure dependence of thermal conductivities of large mass ratio compounds. Phys. Rev. B 91, 121202 (2015).

103. Ma, J., Li, W. \& Luo, X. Intrinsic thermal conductivity and its anisotropy of wurtzite InN. Appl. Phys. Lett. 105, 082103 (2014).

104. Qin, G. et al. Anisotropic intrinsic lattice thermal conductivity of phosphorene from first principles. Phys. Chem. Chem. Phys. 17, 4854-4858 (2015).

105. Zhu, L., Zhang, G. \& Li, B. Coexistence of size-dependent and size-independent thermal conductivities in phosphorene. Phys. Rev. B 90, 214302 (2014).

106. Zhang, J. et al. Phosphorene nanoribbon as a promising candidate for thermoelectric applications. Sci. Rep. 4, 6452 (2014).

107. Sanati, M., Albers, R. C., Lookman, T. \& Saxena, A. Elastic constants, phonon density of states, and thermal properties of UO2. Phys. Rev. B 84, 014116 (2011).

108. Yin, Q. \& Savrasov, S. Y. Origin of low thermal conductivity in nuclear fuels. Phys. Rev. Lett. 100, 225504 (2008).

109. Yun, Y., Legut, D. \& Oppeneer, P. M. Phonon spectrum, thermal expansion and heat capacity of UO2 from first-principles. J. Nucl. Mater. 426, 109-114 (2012)

110. Dolling, G., Cowley, R. A. \& Woods, A. D. B. Crystal dynamics of uranium dioxide. Can. J. Phys. 43, 1397-1413 (1965).

111. Lodziana, Z. \& Parliński, K. Dynamical stability of the alpha and theta phases of alumina. Phys. Rev. B 67, 174106 (2003).

112. Schober, H., Strauch, D. \& Dorner, B. Lattice dynamics of aapphire (Al2O3). Z. Phys. B Condens. Mat. 92, 273-283 (1993).

113. Hector, L. G., Herbst, J. F. \& Kresse, G. Ab Initio thermodynamic and elastic properties of alkaline-earth metals and their hydrides. Phys. Rev. B 76, 014121 (2007)

114. Hu, C. H. et al. Crystal structure prediction of $\mathrm{LiBeH} 3$ using ab initio total-energy calculations and evolutionary simulations. J. Chem. Phys. 129, 234105 (2008)

115. Wdowik, U. D. \& Parliński, K. Lattice dynamics of cobalt-deficient CoO from first principles. Phys. Rev. B 78, 224114 (2008).
116. Derzsi, M. et al. Effects of Coulomb interaction on the electronic structure and lattice dynamics of the Mott insulator Fe2SiO4 spinel. Phys. Rev. B 79 , 205105 (2009).

117. Duan, Y. H. \& Sorescu, D. C. Density functional theory studies of the structural, electronic, and phonon properties of $\mathrm{Li} 2 \mathrm{O}$ and $\mathrm{Li} 2 \mathrm{CO} 3$ : application to $\mathrm{CO} 2$ capture reaction. Phys. Rev. B 79, 014301 (2009).

118. Evarestov, R. A. \& Losev, M. V. All-electron LCAO calculations of the LiF crystal phonon spectrum: influence of the basis set, the exchange-correlation functional, and the supercell size. J. Comput. Chem. 30, 2645-2655 (2009).

119. Jiang, C., Stanek, C. R., Marks, N. A., Sickafus, K. E. \& Uberuaga, B. P. Predicting from first principles the chemical evolution of crystalline compounds due to radioactive decay: the case of the transformation of $\mathrm{CsCl}$ to $\mathrm{BaCl}$. Phys. Rev. B 79, 132110 (2009).

120. Luo, X. H., Zhou, W., Ushakov, S. V., Navrotsky, A. \& Demkov, A. A. Monoclinic to tetragonal transformations in hafnia and zirconia: A combined calorimetric and density functional study. Phys. Rev. B 80, 134119 (2009).

121. Minamoto, S., Kato, M., Konashi, K. \& Kawazoe, Y. Calculations of thermodynamic properties of $\mathrm{PuO} 2$ by the first-principles and lattice vibration. J. Nucl. Mater. 385, 18-20 (2009).

122. Sevik, C. \& Cagin, T. Mechanical and electronic properties of $\mathrm{CeO} 2, \mathrm{ThO} 2$, and (Ce,Th)O-2 alloys. Phys. Rev. B 80, 014108 (2009).

123. Shi, S. Q. et al. First-principles investigation of the bonding, optical and lattice dynamical properties of CeO2. J. Power Sources 194, 830-834 (2009).

124. Shi, S. Q. et al. First-principles study of lattice dynamics of LiFePO4. Phys. Lett. A 373, 4096-4100 (2009).

125. Wdowik, U. D. \& Legut, D. Ab initio lattice dynamics of MnO. J. Phys. Condes. Matter 21, 275402 (2009).

126. Wrobel, J., Kurzydlowski, K. J., Hummer, K., Kresse, G. \& Piechota, J. Calculations of $\mathrm{ZnO}$ properties using the Heyd-Scuseria-Ernzerhof screened hybrid density functional. Phys. Rev. B 80, 155124 (2009).

127. Wdowik, U. D. Structural stability and thermal properties of BeO from the quasiharmonic approximation. J. Phys. Condes. Matter 22, 045404 (2010).

128. Wei, L. et al. Lattice dynamics of bismuth-deficient BiFeO 3 from first principles. Comput. Mater. Sci. 111, 374-379 (2016).

129. Wang, Y., Liu, Z. K., Chen, L. Q., Burakovsky, L. \& Ahuja, R. First-principles calculations on MgO: Phonon theory versus mean-field potential approach. J. Appl. Phys. 100, 023533 (2006)

130. Kleinman, L. \& Bylander, D. M. Efficacious form for model pseudopotentials. Phys. Rev. Lett. 48, 1425-1428 (1982).

131. Gonze, X., Charlier, J. C., Allan, D. C. \& Teter, M. P. Interatomic force-constants from first principles-the case of alpha-quartz. Phys. Rev. B 50, 13035-13038 (1994).

(i) This work is licensed under a Creative Commons Attribution 4.0 article are included in the article's Creative Commons license, unless indicated otherwise in the credit line; if the material is not included under the Creative Commons license, users will need to obtain permission from the license holder to reproduce the material. To view a copy of this license, visit http://creativecommons.org/licenses/ by/4.0/ 